\title{
BMJ Open Rationale and design of a prospective, multicentre, randomised, conventional treatment-controlled, parallel-group trial to evaluate the efficacy and safety of ulinastatin in preventing acute respiratory distress syndrome in high- risk patients
}

\author{
Zongyu Wang, ${ }^{1}$ Liyuan Tao, ${ }^{2}$ Yingying Yan, ${ }^{3}$ Xi Zhu ${ }^{1}$
}

To cite: Wang Z, Tao L, Yan Y, et al. Rationale and design of a prospective, multicentre, randomised, conventional treatment-controlled, parallelgroup trial to evaluate the efficacy and safety of ulinastatin in preventing acute respiratory distress syndrome in high-risk patients. BMJ Open 2019;9:e025523. doi:10.1136/ bmjopen-2018-025523

- Prepublication history for this paper is available online To view these files, please visit the journal online (http://dx.doi. org/10.1136/bmjopen-2018025523).

Received 19 July 2018 Revised 6 January 2019 Accepted 7 February 2019

Check for updates

(C) Author(s) (or their employer(s)) 2019. Re-use permitted under CC BY-NC. No commercial re-use. See rights and permissions. Published by BMJ.

For numbered affiliations see end of article.

Correspondence to

Professor Xi Zhu;

xizhuccm@163.com

\section{ABSTRACT}

Introduction Acute respiratory distress syndrome (ARDS) is challenging in the intensive care unit (ICU). Although pharmacotherapy for ARDS has gained increasing attention, most trials have yielded negative results. Patients with ARDS have usually been recruited as subjects; the inflammatory reaction has already expanded into a cascade at this point, and its severity is sufficient to damage the lung parenchyma. This raises the question of whether early treatment can prevent ARDS and the associated lung injury. We hypothesise that ARDS is preventable in high-risk patients by administration of ulinastatin as an anti-inflammatory drug before ARDS onset, and we are performing a study to test ulinastatin, a protease inhibitor, versus treatment-as-usual in a group of patients at increased risk for ARDS.

Methods and analysis This report presents the protocol for a multicentre, randomised, conventional treatment-controlled, parallel group study to prevent the development of ARDS using ulinastatin in high-risk patients. The study population will comprise patients at risk of ARDS in the ICU ( $\geq 18$ years of age and Lung Injury Prediction Score of $>4)$; patients with confirmed ARDS and some other conditions (immunodeficiency, use of some drugs, etc.) will be excluded. The enrolled patients will be randomly allocated to an ulinastatin group (ulinastatin will be intravenously administered every 8 hours for a total of $600000 \mathrm{U} /$ day for five consecutive days) or control group. The efficacy of ulinastatin in preventing ARDS development will be evaluated by the incidence rate of ARDS as the primary outcome; the secondary outcomes include the severity of ARDS, clinical outcome, extrapulmonary organ function and adverse events incurred by ulinastatin. Based on the results of preliminary studies and presuming the incidence of ARDS will decrease by $9 \%$ in high-risk patients, 880 patients are needed to obtain statistical power of $80 \%$.

Ethics and dissemination This study has been approved by the Peking University Third Hospital Medical Science Research Ethics Committee. The findings will be published
Strengths and limitations of this study

- This is the first multicentre randomised controlled study to evaluate the prophylactic effect of ulinastatin on acute respiratory distress syndrome in an anti-inflammatory intervention perspective with the potential to provide a drug candidate for future prevention.

- A widely used screening tool is being adopted to weight risk factors with the aim of sorting out the given study population as accurately as possible.

- Allocation concealment is not being adopted, which is a limitation of this study.

- The sources of potential bias and imprecision could limit the validity and generalisability of the study; one such source is the lack of comparability of predefined endpoints among participating hospitals.

in peer-reviewed journals and presented at national and international conferences.

Trial registration number NCT03089957; Pre-results.

\section{BACKGROUND}

Acute respiratory distress syndrome (ARDS) is devastating to critically ill patients in the intensive care unit (ICU). Although the incidence of ARDS is decreasing, ${ }^{1}$ the mortality rate of moderate and severe ARDS exceeds $40 \%{ }^{2}$ Various strategies and therapeutic measures, such as lung-protective mechanical ventilation, ${ }^{3} 4$ early neuromuscular blockade, ${ }^{5}$ and a conservative fluid strategy, ${ }^{6}$ have been considered to improve the prognosis or increase the ventilator-free days in patients with ARDS. However, the management of ARDS still needs improvement, and 
no effective interventions that target the pathogenesis have been established. Statins, inhaled nitric oxide and keratinocyte growth factor, once considered beneficial in patients with ARDS, were not confirmed to improve outcomes in randomised controlled clinical trials. ${ }^{7-10}$

Neutrophils and inflammatory mediators act directly on the vascular endothelium and alveolar epithelium, leading to inflammatory lung injury. ${ }^{11}$ After initiation, the inflammation progresses in a cascade. Application of pharmacological therapies that target the key aspects of the pathogenesis of ARDS at the inappropriate time is worthy of attention; however, it is difficult to impede the developing inflammation, which probably accounts for the disappointing results in part. Most previous clinical trials enrolled patients with ARDS as subjects, and the inflammation had progressed to an uncontrollable stage at this point. ${ }^{712-14}$

Consequently, a shift in focus from treatment to prevention of ARDS is of value. ${ }^{15} 16$ Trials designed to prevent ARDS have attracted the attention of investigators because the expanding inflammation might be restrained before becoming irremediable. Patients at risk of developing ARDS are abundant in clinical practice. A prospective survey of 3023 patients in the ICU showed that about one-third of patients receiving mechanical ventilation were at risk of ARDS, pulmonary complications occurred frequently and clinical outcomes were worse than in patients not at risk. ${ }^{17}$ Emergency department-based studies have revealed sepsis-associated ARDS progression rates after ICU admission ranging from $8.9 \%$ to $27.5 \% .^{18}{ }^{19}$ Therefore, prevention of ARDS in at-risk patients is essential.

Inflammatory lung injury plays a major role in the pathogenesis of ARDS. ${ }^{20}$ Calfee et al found that the hyperinflammatory subphenotype of ARDS was characterised by more severe shock, metabolic acidosis and worse clinical outcomes. ${ }^{21}$ Some promising approaches, including generic pharmacological compounds, combination therapies and cell-based therapies, have also been evaluated with respect to their potential to cure ARDS. ${ }^{22}$ Ulinastatin is a pharmacological compound that acts as a broad-spectrum protease inhibitor of serine proteinase, neutrophil elastase and other enzymes. It specialises in halting destruction of the lung parenchyma, and its use is considered part of the anti-inflammatory strategy for various diseases including acute pancreatitis and the cardiopulmonary bypass-related inflammatory response. ${ }^{23} 24$ The use of ulinastatin has been explored in animal experiments of lung injury as well. ${ }^{25}{ }^{26}$ The anti-inflammatory property of ulinastatin and its protective effect have been shown in both experimental and clinical studies. However, one systematic review and meta-analysis showed that high-quality original research of the use of ulinastatin for ARDS is scarce; of all 29 studies evaluated, 4 had a Jadad score of 2 and 25 had a Jadad score of $1 .^{27}$ Additionally, the clinical data on chemoprophylaxis of ARDS have yielded negative results so far. Therefore, whether the onset of ARDS can be stopped by ulinastatin remains

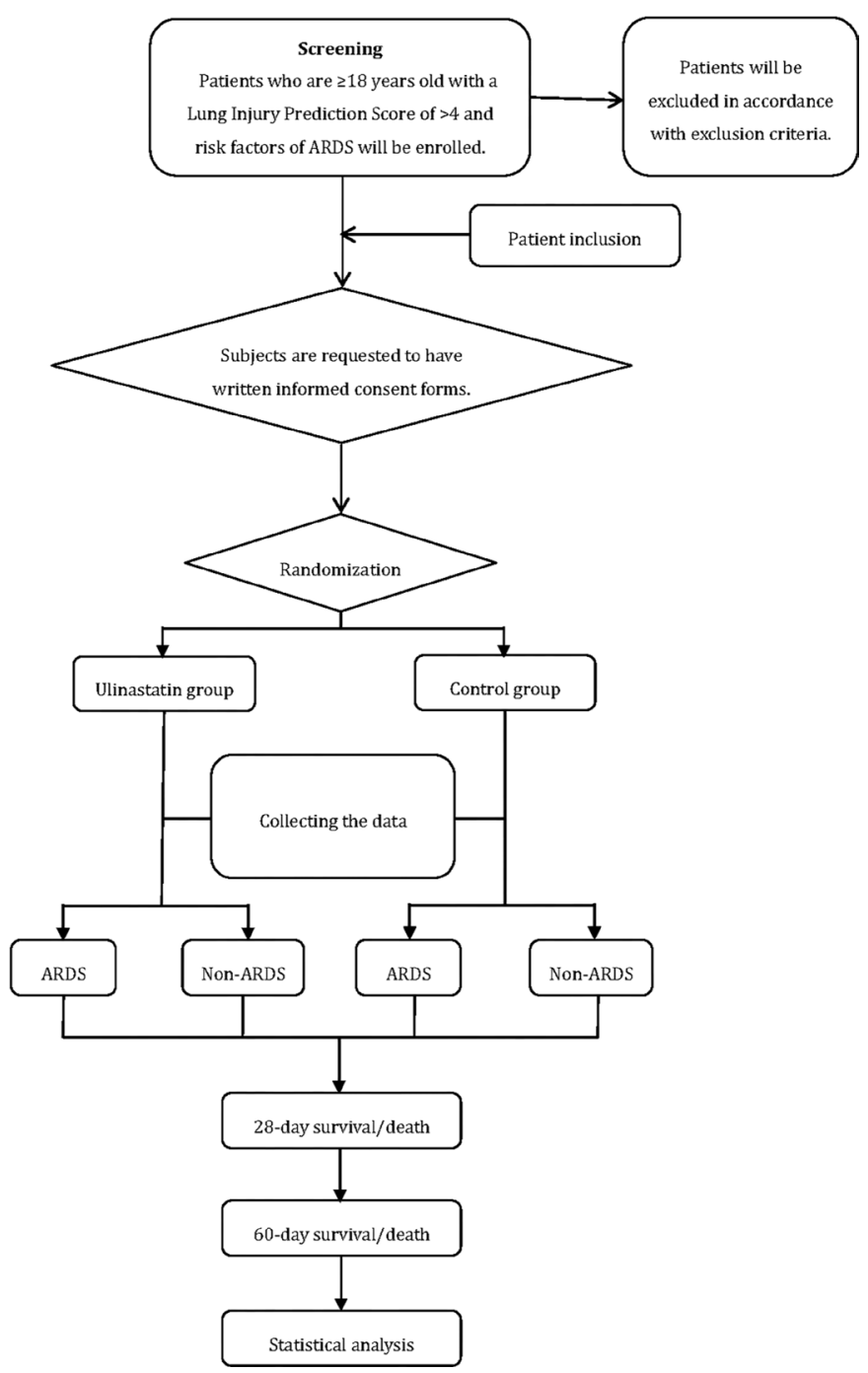

Figure 1 Study flow chart. ARDS, acute respiratory distress syndrome.

unclear. We intend to fill this knowledge gap through a randomised controlled parallel-group trial. The evidence needs to be accumulated sequentially to seek an alternative option to reduce the burden of ARDS.

In the present study, we will examine the effectiveness of ulinastatin to prevent the development of ARDS in high-risk patients and measure its effects on the severity and outcomes of ARDS. We will also assess the safety of ulinastatin.

\section{METHODS AND ANALYSIS \\ Study design}

This study is a multicentre, randomised, controlled, parallel-group clinical trial that is being implemented in eight centres in China. All centres are ICU departments in university hospitals or local general hospitals. The study process is presented in figure 1 . Patients who are $\geq 18$ years old with a Lung Injury Prediction Score (LIPS) of $>4$ and any of the following risk factors will be enrolled: bacteraemia, septic shock, pneumonia, 
multiple fractures, lung contusion, aspiration, recurrent transfusion, or severe acute pancreatitis. Patients with any one of the following conditions will be excluded: a diagnosis of ARDS; HIV infection; therapy with a cytotoxic drug; neutrophilic granulocytopenia (except secondary to sepsis); another immunodeficiency (eg, leukaemia); a history of solid organ or bone marrow transplantation; chronic pulmonary disease (except chronic obstructive pulmonary diseases and asthma); vasculitis; use of granulocyte/granulocyte-macrophage colony-stimulating factor, aspirin, clopidogrel, hormones at more than the physiological replacement dose, thymosin or gamma globulin, and Xuebijing injection within 1 month; participation in another trial within the past 3 months; pregnancy or lactation; or discontinuation of treatment.

The LIPS, formulated by Gajic et al, is used to gauge predisposing factors and risk modifiers; different points are assigned to these factors and modifiers, and the sum of all points is the LIPS. ${ }^{28}$ This score is used as a primary element to determine patient enrolment suitability. The diagnosis of ARDS will be determined by the Berlin definition. $^{20}$

The enrolled patients will be randomly divided into two groups, the ulinastatin group and control group, using unified blocked randomisation codes produced using SAS V.9.1 at the Research Center of Clinical Epidemiology, Peking University Third Hospital (PUTH). The sealed envelope method will be used for allocation concealment. The envelopes will be distributed to the research units and maintained by a specially appointed person. Random assignment will be conducted within each centre. The random numbers in each envelope will determine which group to which the patients are assigned. The intervention in each group will not begin until the grouping result is known. Data analysts and statistical personnel will be blinded to avoid the bias resulting from subjective impression.

\section{Intervention and follow-up}

The patients in the ulinastatin group will be intravenously administered $200000 \mathrm{U}$ of ulinastatin dissolved in $100 \mathrm{~mL}$ of saline. The infusion will be finished within 1 hour. This ulinastatin administration will be repeated at 8-hour intervals for a total of $600000 \mathrm{U} /$ day for five consecutive days. The patients in the control group will receive conventional therapy only. Considering that most of the enrolled patients will be critically ill and require mechanical ventilation and sedation, drug therapy will not be regarded as a main interference factor on the findings, and ulinastatin will not be disguised in the study. Insufficient funding support for this study is another reason that the patients and investigators will not be blinded to the intervention. However, the medical personnel responsible for collecting the research data will be blinded to offset the bias brought about by lack of allocation concealment. The actual dose of ulinastatin during drug use will be recorded in detail to achieve deep analysis. Additionally, both the ulinastatin group and control group will receive identical fundamental treatment. Routine mechanical ventilation management will be administered to patients with ARDS according to the recommendations of evidence-based clinical practice guidelines. ${ }^{29}$

Patients will be followed up until the 60th day after random allocation or death. At least two follow-up visits will be implemented to collect survival data (on days 28 and 60 , respectively). The first visit will be performed to collect the available predefined outcome data, and the second will be performed to collect all primary and secondary outcome data.

\section{Indicators}

The primary objective will be to determine whether prophylactic ulinastatin in patients at high risk for developing ARDS reduces the incidence of subsequent ARDS versus conventional treatment. For this purpose, all patients will be examined for the development of ARDS once a day for 7 days, or until discharge from the ICU or death. The secondary objectives will be the differences in the following parameters between the two groups:

- Severity of ARDS.

- Clinical outcome at 28 and 60 days.

- Proportions of patients receiving invasive/non-invasive mechanical ventilation.

- Duration of mechanical ventilation.

- Morbidity of extrapulmonary organ dysfunction (coagulation, liver, circulatory system, consciousness, or kidney dysfunction).

- Duration of stay in ICU and hospital.

- Medical expense.

- Drug-related adverse events.

The severity of ARDS will be measured by the ratio of arterial oxygen $\mathrm{PaO} 2$ to fractional inspired oxygen. The clinical outcome will be whether a patient is still alive at 28 and 60 days. Morbidity of extrapulmonary organ dysfunction will be defined as the proportion of concurrent organ dysfunction aside from lung dysfunction as assessed by organ-specific indicators. The medical expense will be defined as the patient's total cost during the hospital stay.

Baseline assessment will involve demographics, medical history, the LIPS and the Acute Physiology and Chronic Health Evaluation II (APACHE II) score. Regular assessment will include the Sequential Organ Failure Assessment score, vital signs, liquid balance, haemodynamics, respiratory mechanics and laboratory indexes.

The safety evaluation of ulinastatin will include adverse drug events (ADEs), serious adverse event (SAEs) and the incidences of these events. ADEs of ulinastatin mainly manifest as an anaphylactic reaction; leucopenia or eosinophilia (haematological system); nausea, vomiting, diarrhoea or elevated liver enzymes (digestive system); or tenderness, redness, itching, or rash at the injection site. SAEs will be defined as any untoward medical occurrence at any dose of ulinastatin that meets one or more of the following criteria: results in death, is life-threatening, causes prolongation of hospitalisation, results in 
persistent or significant disability/incapacity, or requires intervention to prevent permanent impairment or damage. All ADEs and SAEs will be recorded, reported and followed up until recovery.

All patients who withdraw from the study will be documented, including the date of and reason for withdrawal. We will also keep a record of violations of the study protocol for late-term analysis.

\section{Statistical analysis}

We intend to enrol 840 patients (approximately 420 patients per group) in this multicentre clinical trial. Eight centres in China are participating in the study, and each centre is enrolling about 120 patients. A pilot study in the ICU of PUTH showed that the incidence of ARDS in high-risk patients was $27 \%$ which is similar to that of an earlier report from ICUs in mainland China. ${ }^{30}$ Another preliminary test of ours indicated that the incidence of ARDS decreased to $18 \%$ in at-risk patients treated with $600000 \mathrm{U}$ of ulinastatin per day. The sample size of the trial was calculated with PASS V.11.0 (NCSS Statistical Software, Kaysville, Utah, USA). Based on the above results and establishment of an equal sample size between the two groups, a two-sided $\mathrm{Z}$ test $(\alpha=0.05, \beta=0.20)$ was used, resulting in 335 patients in each group. Given a $20 \%$ default rate, 420 patients each are needed for the intervention and control groups (840 patients in total). For convenient calculation, each centre will provide 110 patients (ie, 55 patients in each of the two groups), and a total of 880 patients will be enrolled in the trial.

The efficacy analysis will be based on an intention-totreat method. The $\chi^{2}$ test will be employed to evaluate the effectiveness and safety of pharmacological prevention. Most secondary outcome analyses will adopt the strategy of the primary outcome analysis. The objects will be divided into subgroups according to the LIPS, and a further subgroup analysis will be performed. A KaplanMeier curve will be used to simulate the cumulative risk of death on days 28 and 60 during follow-up. Log-rank tests will be used to compare survival distributions. A Cox proportional hazard model will be used to calculate hazard proportions and 95\% CIs. We will use multivariate logistic regression to analyse the factors influencing the onset of ARDS, and clinical variables such as age, sex, type of ARDS and APACHE II score will be applied to the analysis and adjustment of the model. In the univariate analysis, we will compare continuous variables using the $\mathrm{t}$ test or Wilcoxon rank sum test and analysis of variance and will compare proportions with the $\chi^{2}$ test or Fisher's exact test, as appropriate.

\section{Study organisation}

This study of the prevention of ARDS with ulinastatin will be conducted by an organisation that is led by PUTH and partnered with several general hospitals in China. The data will be converged from all the centres, and the analysis will be performed independently by statistical experts at the Research Centre of Clinical Epidemiology in PUTH.

To ensure the study quality, adherence to the protocol and patient safety, a board composed of academic members and an independent statistician will regularly monitor the eligible patients, data correctness and integrity, and progress of the study through on-site inspection or online checks (electronic case report forms compiled by a data-collection system). The researchers in all the centres will be required to undergo unified training to record the data and diagnoses, and they will be required to fill in the case report form truthfully and in detail. The observation and discovery of the patients will be verified to ensure that all clinical conclusions are based on raw data. An interim analysis will be scheduled at the time of recruiting half of the patients, and the results of this analysis will remain unknown to the direct participants of the study. The following situations will result in premature termination of this study: the occurrence of serious safety events, exposure of a key flaw in the protocol, serious deviation in programme delivery and a request for termination by the leading investigators. An open and constant communication channel will be maintained throughout the course of the study. The investigators will regularly hold face-to-face meeting to discuss problems and offer solutions. Communication will also occur by other appropriate methods, such as Web conferencing and social software.

\section{Ethics and dissemination}

The study is being carried out according to the ethical principles proclaimed in the 1964 Declaration of Helsinki, revised in Tokyo in 2004. The study information will be provided to all patients, and written informed consent will be obtained from each individual patient or his/her next of kin before being included in the study. The results of the study will be presented in national and international conferences and published in peer-reviewed journals. This study protocol has been written in accordance with the Standard Protocol Items: Recommendations for Interventional Trials 2013 statement.

\section{Patient and public involvement}

Some special conditions that exist in the ICU, such as critical illness, poor communication with tracheal intubation, sedation, the relatively closed environment and others are unfavourable for patient and public involvement. Instead, the present study only has direct implications for the medical staff. Therefore, there is no reason to include patients or the public in this study as a symbolic act.

\section{DISCUSSION}

As one of the inclusion criteria, the LIPS will be employed to screen all candidates in the study. The LIPS was introduced in 2011 and is composed of predisposing conditions and risk modifiers. It is a validated prediction model to identify patients at high risk for ARDS. ${ }^{28}$ With respect to 
predictors, the LIPS is extremely similar to another acute LIPS published in the same year ${ }^{31}$; however, the LIPS has been a more frequently adopted scoring model in clinical research. ${ }^{32-34}$ The construction of a forecasting model prompts the hypothesis that ARDS is preventable to some extent. Other scoring methods have also been proposed, such as surgical lung injury prediction, surgical lung injury prediction-2 and early acute lung injury, applying to specially targeted surgical populations with critical illness or detecting early ARDS prior to the need for mechanical ventilation. ${ }^{35-37}$ The LIPS has been shown to predict ARDS with unsatisfactory sensitivity and specificity, and further refinements were needed. ${ }^{17}$ Nevertheless, we have selected the LIPS as one of the screening criteria for the present study because it was derived from and validated in a large-sample cohort and has been tested in various populations, including non-emergency department hospitalised patients and critically ill surgical patients. ${ }^{38} 39$ In addition, evidence is available to support the LIPS for assessment of the independent risk of ARDS in patients with severe sepsis or receiving mechanical ventilation in the emergency department. ${ }^{18} 40$ Admittedly, use of the LIPS has not yet been confirmed in critically ill patients in the ICU setting. However, the aforementioned populations actually cover ICU admission, and the applicability of the LIPS in the ICU has been indirectly guaranteed.

Ulinastatin is a glycoprotein extracted from human urine and acts as a serine protease inhibitor with anti-inflammatory activity. It is a frequently used therapeutic agent that has shown positive effects. In one randomised controlled trial, ulinastatin was prescribed to patients with severe sepsis, and the study findings suggested a reduced mortality rate in the modified intention-to-treat analysis. ${ }^{41}$ Ulinastatin is regarded as a promising candidate therapy for sepsis. ${ }^{42}$ Targeted prophylaxis of ARDS in high-risk patients has begun to attract the attention of clinicians and researchers, but no previously reported studies have evaluated ulinastatin as a drug candidate. Ulinastatin is being used for the first time to prevent the development of ARDS in a high-risk group of patients who are susceptible to ARDS in the present randomised controlled trial, which is the main highlight of this study. This is also where we hope to make an improvement over previous correlational studies.

The LIPS is used to stratify the risk of ARDS among patients admitted to the ICU; thus, the target population of the present study is appropriate. Ulinastatin will be administered to block the initial stage of the inflammatory process and avoid lung parenchymal damage and eventual destruction. We will then carry out our exploration of the feasibility of this drug.

This study has some limitations. The test drug will not be concealed, which will introduce assessment and expectation biases. Moreover, the sources of potential bias and imprecision could limit the validity and generalisability of the results; such sources of bias include the lack of comparability of predefined endpoints, such as medical expense, among the participating hospitals.

\section{CONCLUSION}

The primary objective of this prospective, multicentre, randomised controlled trial is to validate the efficacy of ulinastatin in the prevention of ARDS in patients at risk of ARDS. The study will also explore secondary outcomes including the severity of ARDS, mortality rate, safety of ulinastatin and other parameters. Eight centres in China are participating in the trial so far, and the first patients were enrolled in early 2017. The participation of the centres will provide enough eligible patients to conduct the trial, diversify the causes of ARDS and improve the representation of the study results. The study design will allow for a robust assessment of the precautions and early pharmacological therapy of ARDS.

\section{Author affiliations}

${ }^{1}$ Department of Intensive Care Unit, Peking University Third Hospital, Beijing, China ${ }^{2}$ Research Center of Clinical Epidemiology, Peking University Third Hospital, Beijing, China

${ }^{3}$ Department of Pharmacy, Peking University Third Hospital, Beijing, China

Acknowledgements The authors appreciate the contributions of Hong-Liang $\mathrm{Li}$ in the study protocol design and Hua Zhang in construction of the online database. The authors also thank Zhao-Zhong Zhu for his helpful comments on the manuscript.

Contributors XZ and ZW conceived and designed the study. ZW wrote the article and provided critical revisions that are important for the intellectual content. LT provided statistical expertise. YY contributed to the clinical registration. All authors approved the final version of the article.

Funding The study was initiated by Peking University Third Hospital. All of the research units will organize self-dependently, participate voluntarily without funding support, and strive to complete the study. We are currently applying for government funding support.

Competing interests None declared.

Patient consent for publication Next of kin consent obtained.

Ethics approval The study protocol and informed consent have been reviewed by the Peking University Third Hospital Medical Science Research Ethics Committee (M2016190) and were approved on 17 February 2017; they were also accepted by each participating centre.

Provenance and peer review Not commissioned; externally peer reviewed.

Open access This is an open access article distributed in accordance with the Creative Commons Attribution Non Commercial (CC BY-NC 4.0) license, which permits others to distribute, remix, adapt, build upon this work non-commercially, and license their derivative works on different terms, provided the original work is properly cited, appropriate credit is given, any changes made indicated, and the use is non-commercial. See: http://creativecommons.org/licenses/by-nc/4.0/.

\section{REFERENCES}

1. Li G, Malinchoc M, Cartin-Ceba R, et al. Eight-year trend of acute respiratory distress syndrome: a population-based study in Olmsted County, Minnesota. Am J Respir Crit Care Med 2011;183:59-66.

2. Villar J, Blanco J, Kacmarek RM. Current incidence and outcome of the acute respiratory distress syndrome. Curr Opin Crit Care 2016;22:1-6.

3. Brower RG, Matthay MA, Morris A, et al. Ventilation with lower tidal volumes as compared with traditional tidal volumes for acute lung injury and the acute respiratory distress syndrome. $N$ Engl J Med 2000;342:1301-8.

4. Brower RG, Lanken PN, Maclntyre N, et al. Higher versus lower positive end-expiratory pressures in patients with the acute respiratory distress syndrome. N Engl J Med 2004;351:327-36.

5. Papazian L, Forel JM, Gacouin A, et al. Neuromuscular blockers in early acute respiratory distress syndrome. $N$ Engl J Med 2010;363:1107-16. 
6. Wiedemann HP, Wheeler AP, Bernard GR, et al. Comparison of two fluid-management strategies in acute lung injury. $N$ Engl $J$ Med 2006;354:909-75.

7. McAuley DF, Laffey JG, O'Kane CM, et al. Simvastatin in the acute respiratory distress syndrome. N Engl J Med 2014;371:1695-703.

8. Truwit JD, Bernard GR, Steingrub J, et al. Rosuvastatin for sepsisassociated acute respiratory distress syndrome. N Engl J Med 2014;370:2191-200.

9. Adhikari NK, Dellinger RP, Lundin S, et al. Inhaled nitric oxide does not reduce mortality in patients with acute respiratory distress syndrome regardless of severity: systematic review and metaanalysis. Crit Care Med 2014;42:404-12.

10. McAuley DF, Cross LM, Hamid U, et al. Keratinocyte growth factor for the treatment of the acute respiratory distress syndrome (KARE): a randomised, double-blind, placebo-controlled phase 2 trial. Lancet Respir Med 2017;5:484-91.

11. Matthay MA, Zemans RL. The acute respiratory distress syndrome: pathogenesis and treatment. Annu Rev Pathol 2011;6:147-63.

12. Taylor RW, Zimmerman JL, Dellinger RP, et al. Low-dose inhaled nitric oxide in patients with acute lung injury: a randomized controlled trial. JAMA 2004;291:1603-9.

13. Matthay MA, Brower RG, Carson S, et al. Randomized, placebocontrolled clinical trial of an aerosolized $\beta_{2}$-agonist for treatment of acute lung injury. Am J Respir Crit Care Med 2011;184:561-8.

14. Zeiher BG, Artigas A, Vincent JL, et al. Neutrophil elastase inhibition in acute lung injury: results of the STRIVE study. Crit Care Med 2004;32:1695-702

15. Yadav H, Thompson BT, Gajic O. Fifty Years of Research in ARDS. Is Acute Respiratory Distress Syndrome a Preventable Disease? Am J Respir Crit Care Med 2017;195:725-36.

16. Gong MN, Thompson BT. Acute respiratory distress syndrome: shifting the emphasis from treatment to prevention. Curr Opin Crit Care 2016;22:21-37.

17. Neto AS, Barbas CSV, Simonis FD, et al. Epidemiological characteristics, practice of ventilation, and clinical outcome in patients at risk of acute respiratory distress syndrome in intensive care units from 16 countries (PRoVENT): an international, multicentre, prospective study. Lancet Respir Med 2016;4:882-93.

18. Mikkelsen ME, Shah CV, Meyer NJ, et al. The epidemiology of acute respiratory distress syndrome in patients presenting to the emergency department with severe sepsis. Shock 2013;40:375-81.

19. Fuller BM, Mohr NM, Dettmer M, et al. Mechanical ventilation and acute lung injury in emergency department patients with severe sepsis and septic shock: an observational study. Acad Emerg Med 2013;20:659-69.

20. Ranieri VM, Rubenfeld GD, Thompson BT, et al. Acute respiratory distress syndrome: the Berlin Definition. JAMA 2012;307:2526-33.

21. Calfee CS, Delucchi K, Parsons PE, et al. Subphenotypes in acute respiratory distress syndrome: latent class analysis of data from two randomised controlled trials. Lancet Respir Med 2014;2:611-20.

22. Matthay MA, McAuley DF, Ware LB. Clinical trials in acute respiratory distress syndrome: challenges and opportunities. Lancet Respir Med 2017;5:524-34.

23. Wang LZ, Luo MY, Zhang JS, et al. Effect of ulinastatin on serum inflammatory factors in Asian patients with acute pancreatitis before and after treatment: a meta-analysis. Int J Clin Pharmacol Ther 2016;54:890-8.

24. Zhang Y, Zeng Z, Cao Y, et al. Effect of urinary protease inhibitor (ulinastatin) on cardiopulmonary bypass: a meta-analysis for China and Japan. PLoS One 2014;9:e113973.
25. Bae HB, Jeong CW, Li M, et al. Effects of urinary trypsin inhibitor on lipopolysaccharide-induced acute lung injury in rabbits. Inflammation 2012;35:176-82.

26. Wang L, Huang X, Kong G, et al. Ulinastatin attenuates pulmonary endothelial glycocalyx damage and inhibits endothelial heparanase activity in LPS-induced ARDS. Biochem Biophys Res Commun 2016;478:669-75.

27. Leng $Y X$, Yang SG, Song YH, et al. Ulinastatin for acute lung injury and acute respiratory distress syndrome: a systematic review and meta-analysis. World J Crit Care Med 2014;3:34-41.

28. Gajic O, Dabbagh O, Park PK, et al. Early identification of patients at risk of acute lung injury: evaluation of lung injury prediction score in a multicenter cohort study. Am J Respir Crit Care Med 2011;183:462-70.

29. Fan E, Del Sorbo L, Goligher EC, et al. An Official American Thoracic Society/European Society of Intensive Care Medicine/Society of Critical Care Medicine Clinical Practice Guideline: Mechanical Ventilation in Adult Patients with Acute Respiratory Distress Syndrome. Am J Respir Crit Care Med 2017;195:1253-63.

30. Du B, An Y, Kang Y, et al. Characteristics of critically ill patients in ICUs in mainland China. Crit Care Med 2013:41:84-92.

31. Trillo-Alvarez C, Cartin-Ceba R, Kor DJ, et al. Acute lung injury prediction score: derivation and validation in a population-based sample. Eur Respir J 2011;37:604-9.

32. Festic E, Ortiz-Diaz E, Lee A, et al. Prehospital use of inhaled steroids and incidence of acute lung injury among patients at risk. J Crit Care 2013;28:985-91.

33. Kor DJ, Carter RE, Park PK, et al. Effect of Aspirin on Development of ARDS in At-Risk Patients Presenting to the Emergency Department: The LIPS-A Randomized Clinical Trial. JAMA 2016;315:2406.

34. Festic E, Carr GE, Cartin-Ceba R, et al. Randomized Clinical Trial of a Combination of an Inhaled Corticosteroid and Beta Agonist in Patients at Risk of Developing the Acute Respiratory Distress Syndrome. Crit Care Med 2017;45:798-805.

35. Kor DJ, Warner DO, Alsara A, et al. Derivation and diagnostic accuracy of the surgical lung injury prediction model. Anesthesiology 2011:115:117-28.

36. Kor DJ, Lingineni RK, Gajic O, et al. Predicting risk of postoperative lung injury in high-risk surgical patients: a multicenter cohort study. Anesthesiology 2014;120:1168-81.

37. Levitt JE, Calfee CS, Goldstein BA, et al. Early acute lung injury: criteria for identifying lung injury prior to the need for positive pressure ventilation*. Crit Care Med 2013;41:1929-37.

38. Soto GJ, Kor DJ, Park PK, et al. Lung injury prediction score in hospitalized patients at risk of acute respiratory distress syndrome. Crit Care Med 2016;44:2182-91.

39. Bauman ZM, Gassner MY, Coughlin MA, et al. Lung injury prediction score is useful in predicting acute respiratory distress syndrome and mortality in surgical critical care patients. Crit Care Res Pract 2015;2015:1-8.

40. Fuller BM, Mohr NM, Miller CN, et al. Mechanical ventilation and ARDS in the ED: a multicenter, observational, prospective, crosssectional study. Chest 2015;148:365-74

41. Karnad DR, Bhadade R, Verma PK, et al. Intravenous administration of ulinastatin (human urinary trypsin inhibitor) in severe sepsis: a multicenter randomized controlled study. Intensive Care Med 2014;40:830-8.

42. Linder A, Russell JA. An exciting candidate therapy for sepsis: ulinastatin, a urinary protease inhibitor. Intensive Care Med 2014;40:1164-7. 